\title{
Reactor Antineutrino Flux and Spectrum Measurement at Daya Bay
}

\author{
Fengpeng $\mathrm{An}^{a, *}$, on behalf of the Daya Bay Collaboration \\ ${ }^{a}$ Insititute of Modern Physics, East China University of Science and Technology, \\ Meilong Road 130, Shanghai, China
}

E-mail: anfpeecust.edu.cn

The Daya Bay Reactor Neutrino Experiment uses an array of eight underground detectors to study antineutrinos from six reactor cores at different baselines. Four antineutrino detectors in the two near experimental halls are used for the measurements. In the latest analysis, the reactor antineutrino flux measurement is improved by the reduction in the systematic uncertainty of the neutron detection efficiency. A new measurement of the prompt energy spectrum of reactor antineutrinos with 1958 days of data shows a significant discrepancy in the shape of the spectrum compared with the Huber-Mueller model prediction. The individual inverse beta decay (IBD) yield and spectra of ${ }^{235} \mathrm{U}$ and ${ }^{239} \mathrm{Pu}$ are extracted for the first time from the variations in the IBD prompt energy spectra with fuel evolution.

40th International Conference on High Energy physics - ICHEP2020

July 28 - August 6, 2020

Prague, Czech Republic (virtual meeting)

\footnotetext{
*Speaker
} 


\section{Introduction of the Daya Bay Neutrino Experiment}

The Daya Bay Neutrino Experiment is designed to precisely measure the neutrino mixing angle $\theta_{13}$, using antineutrinos produced in nuclear reactors. In 2012, Daya Bay discovered the non-zero value of $\theta_{13}$ [1]. Besides the main physics goal, the Daya Bay Experiment made precise measurements of reactor antineutrino flux and spectrum. The Daya Bay reactor complex consists of six reactors with total thermal power of $17.4 \mathrm{GW}$, emitting $\sim 3.5 \times 10^{21}$ electron antineutrinos per second. The antineutrinos are produced by the beta decay of fission products of the four primary isotopes: ${ }^{235} \mathrm{U},{ }^{238} \mathrm{U},{ }^{239} \mathrm{Pu}$ and ${ }^{241} \mathrm{Pu}$. Each isotope has a distinct antineutrino spectrum, that can be described by models based on the ILL measurements [2, 3] or ab-initio calculation $[3,4]$. The Daya Bay Neutrino Experiment detects antineutrinos via the inverse beta decay reaction:

$$
\bar{v}_{e}+p \rightarrow e^{+}+n
$$

The produced positron annihilates immediately forming a prompt signal and the neutron is captured on gadolinium or hydrogen, forming a delayed signal. This coincidence signature helps the experiment to effectively suppress the backgrounds.

Eight identically designed antineutrino detectors (ADs) are installed in three experimental halls (EHs). Each detector consists of three zones of cylindrical vessels: the innermost acrylic vessel (IAV) is filled with 20 tons of gadolinium doped liquid scintillator (Gd-LS) serving as the antineutrino target; the middle acrylic vessel is filled with 20 tons of pure scintillator acting as the gamma catcher and the outer stainless steel vessel is filled with 40 tons of mineral oil providing radiation shielding. The combined detection efficiency of the detector is $80.2 \% \pm 1.2 \%$ (including the target proton uncertainty) [6] and the energy resolution is $8.5 \% / \sqrt{E[\mathrm{MeV}]}$.

Figure 1 shows the layout of the Daya Bay Neutrino Experiment, and Figure 2 shows structure of the Daya Bay antineutrino detector.

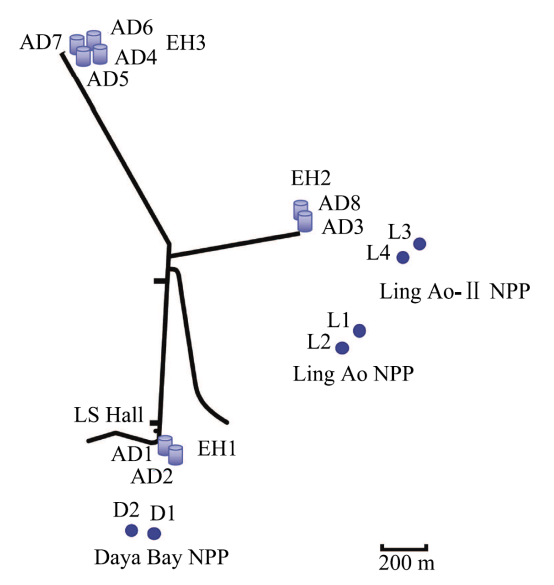

Figure 1: Layout of the full configuration of the Daya Bay experiment. [5]

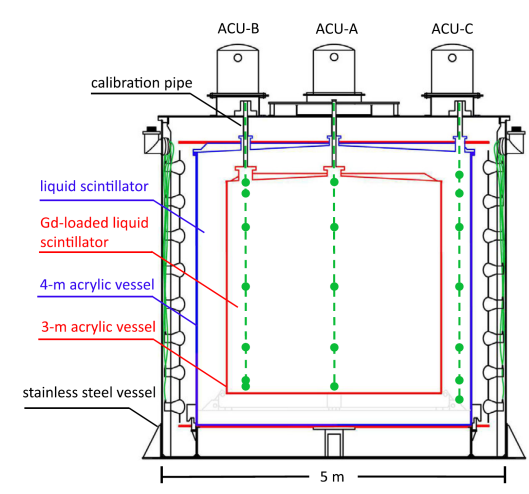

Figure 2: Schematic diagram of the Daya Bay antineutrino detector. [6] 


\section{Recent improvements of the Daya Bay Experiment}

The Daya Bay Neutrino Experiment makes constant efforts to improve its systematic uncertainties. The energy scale uncertainty is improved from $1.0 \%$ to $0.5 \%[7,8]$. The uncertainty of ${ }^{9} \mathrm{Li} /{ }^{8} \mathrm{He}$ background is reduced from $45 \%$ to $30 \%$ [8]. The spent nuclear fuel (SNF) uncertainty is reduced from $100 \%$ to $30 \%$ [8]. A series of efforts were made to improve the neutron detection efficiency uncertainties including comprehensive detector calibrations and model studies. Two radioactive sources, Am-C and Am-Be were deployed in three vertical axis (see Figure 2) inside and outside the Gd-LS region and totally 59 calibration source-location points were made [6]. Five neutron scattering model combinations and four $\mathrm{n}-\mathrm{Gd}$ capture gamma models were considered, and totally 20 simulation model combinations were studied to get a better understanding of the detector performance [6]. With these detailed studies the neutron efficienty uncertainty is improved from $1.69 \%$ to $0.74 \%[6,8]$.

\section{Improved measurement of reactor $\bar{v}_{e}$ flux and spectrum}

With reduced systematic uncertainties and a much larger data sample, the Daya Bay Experiment improves the uncertainties of reactor antineutrino flux and spectrum measurements. Compared with the analysis based on 621 days of data, the uncertainty in the reactor flux measurement is reduced from $2.1 \%$ to $1.5 \%$. The ratio of flux measurement over prediction from the Huber Muller model changed from $0.964 \pm 0.02(\exp$.) to $0.952 \pm 0.014(\exp$.). As shown in Figure 3, the ratio, agrees with the global average with $1 \sigma$.

Based on the 1958 days data sample, the Daya Bay Experiment makes an improved measurement of the reactor antineutrino spectrum [9]. Comparing with Huber-Mueller model prediction, the spectral shape of the measured spectrum disagrees with the prediction with a discrepancy of $5.3 \sigma$ in the entire energy range of $0.7-8 \mathrm{MeV}$, and an excess in the $4 \sim 6 \mathrm{MeV}$ energy range is observed with $6.3 \sigma$ deviation (see Figure 4 ), while it was $4 \sigma$ in the 621 days analysis.

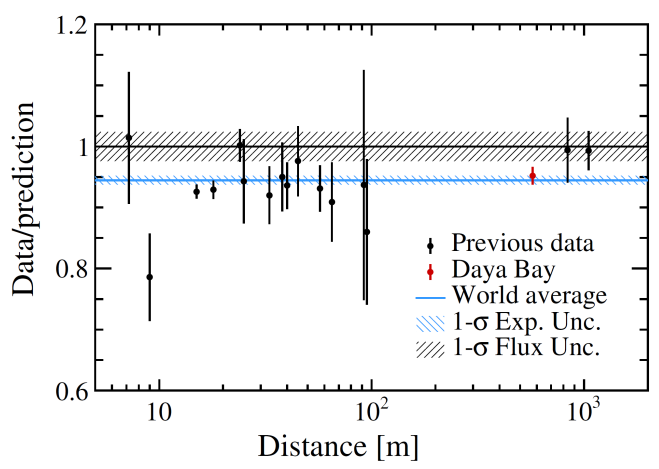

Figure 3: The ratio of measured reactor $\bar{v}_{e}$ yield to the Huber + Mueller model prediction, as a function of reactor-detector distance (1230 days data sample) [6].

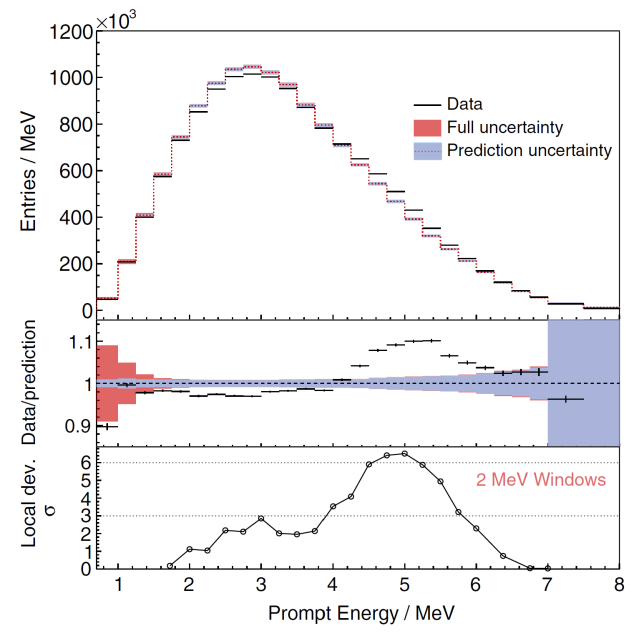

Figure 4: Daya Bay measured and Huber-Mueller model predicted prompt energy spectra (1958 days data sample) [9]. 


\section{Extraction of the ${ }^{235} \mathbf{U}$ and ${ }^{239} \mathbf{P u} \bar{v}_{e}$ spectra at Daya Bay}

The reactors in the Daya Bay Neutrino Experiment are pressurized water reactors (PWR), the initial fissile isotopes of the fresh fuel are ${ }^{235} \mathrm{U}$ and ${ }^{238} \mathrm{U}$, with ongoing reactor burn-up, ${ }^{239} \mathrm{Pu}$ and ${ }^{241} \mathrm{Pu}$ are gradually produced by neutron captures. Figure 5 shows an example of the fission fraction of the four main isotopes in a typical PWR refueling cycle. Figure 6 shows the weekly fission fraction of the major fissile isotopes of all the refueling cycles in the six Daya Bay reactors in 1958 days. As a result of the fission fraction evolution, the reactor antineutrino spectrum evolves with time.

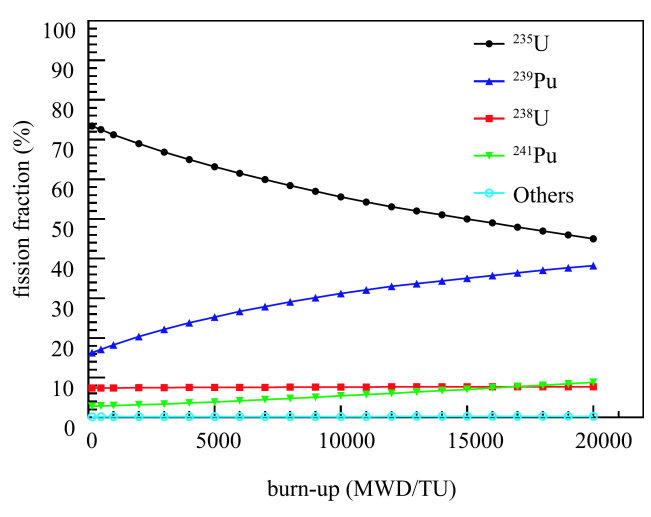

Figure 5: Fission fractions of isotopes in a Daya Bay reactor core as a function of cycle burn-up from a simulation of a typical refueling cycle [5].

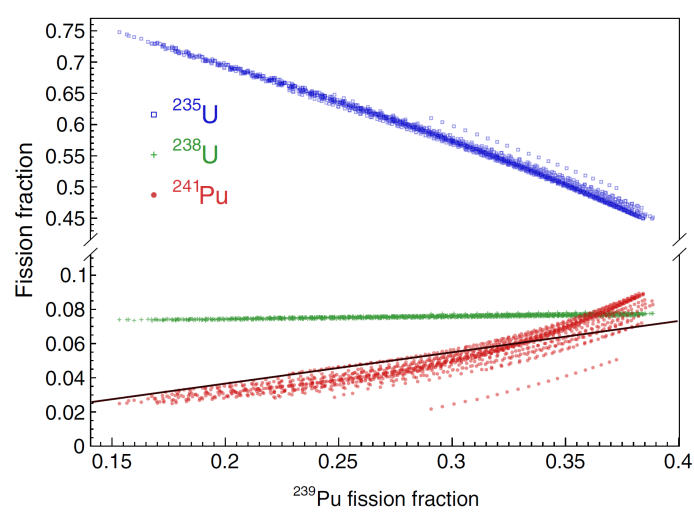

Figure 6: The weekly fission fractions for the four major isotopes in the six reactors in 1958 days of the Daya Bay reactors. The solid line represents the approximately linear relation between ${ }^{239} \mathrm{Pu}$ and ${ }^{241} \mathrm{Pu}$ fission fractions [9].

By using the valuable reactor information provided by the Daya Bay power plant such as the reactor thermal power, the fission fraction of fissile isotopes etc., together with the precisely measured time evolving antineutrino spectrum, the Daya Bay Experiment succeeded in extracting the antineutrino spectrum of ${ }^{235} \mathrm{U}$ and ${ }^{239} \mathrm{Pu}$ [9]. The 3.5 million antineutrino events in the near sites detectors were divided into 20 groups ordered by the effective fission fraction of ${ }^{239} \mathrm{Pu}$, where the effective fission fractions are defined as the fission fractions 'observed' by the detectors of all the 6 reactors $[9,10]$. Figure 7 shows an example of the ${ }^{239} \mathrm{Pu}$ effective fraction of the near sites. The effective fission fraction of ${ }^{235} \mathrm{U}$ ranges from $50 \%$ to $65 \%$, and ${ }^{239} \mathrm{Pu}$ from $24 \%$ to $35 \%$.

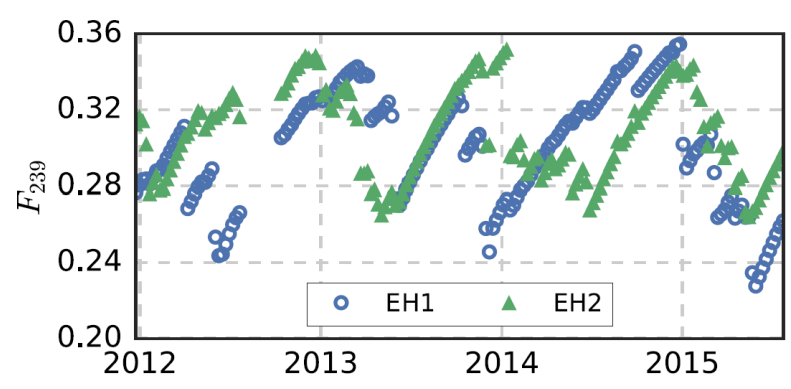

Figure 7: Weekly effective fission fractions of ${ }^{239} \mathrm{Pu}$ for the EH1 and EH2 ADs based on input reactor data $[10]$. 
The extraction of the individual antineutrino spectrum is done by fitting the grouped data by treating ${ }^{235} \mathrm{U}$ and ${ }^{239} \mathrm{Pu}$ spectra as two unknown arrays, each with 26 energy bins, while using the ${ }^{238} \mathrm{U}$ and ${ }^{241} \mathrm{Pu}$ spectra as known priors, since this measurement is not sensitive to the later two isotopes.

Two methods are used to do the fitting. In the first method, a $\chi^{2}$ function is constructed as below:

$$
\chi^{2}\left(\boldsymbol{\eta}_{k}^{5}, \boldsymbol{\eta}_{k}^{9}\right)=2 \sum_{d j k}\left(S_{d j k}-M_{d j k}+M_{d j k} \ln \frac{M_{d j k}}{S_{d j k}}\right)+f(\boldsymbol{\epsilon}, \mathbf{\Sigma})
$$

where $d, j, k$ are the indices of detector, data group, and prompt energy bin, $M_{d j k}$ is the measured prompt energy spectrum of each data group, $S_{d j k}$ is the corresponding expected prompt energy spectrum, $\boldsymbol{\epsilon}$ is a set of nuisance parameters, and $f(\boldsymbol{\epsilon}, \boldsymbol{\Sigma})$ is the term to contrain $\boldsymbol{\epsilon}$ with covariance matrix $\boldsymbol{\Sigma}$.

The energy spectra of ${ }^{238} \mathrm{U}$ and ${ }^{241} \mathrm{Pu}$ are included in the nuisance parameters $\boldsymbol{\epsilon}$, and $>10 \%$ uncertainties are assigned to the two isotopes in both the rate and the shape. The time-dependent terms from non-equilibrium isotopes, spent nuclear fuel, nonlinear nuclides, and backgrounds are considered.

In the second method, a Bayesian inference based Markov Chain Monte Carlo was used to sample the posterior distribution of the antineutrino spectrum of ${ }^{235} \mathrm{U}$ and ${ }^{239} \mathrm{Pu}$, and produced a consistent result with the $\chi^{2}$ fitting results.

Figure 8 shows the extracted ${ }^{235} \mathrm{U}$ and ${ }^{239} \mathrm{Pu}$ prompt energy spectra, which is the first extraction of such spectra of commercial reactors.

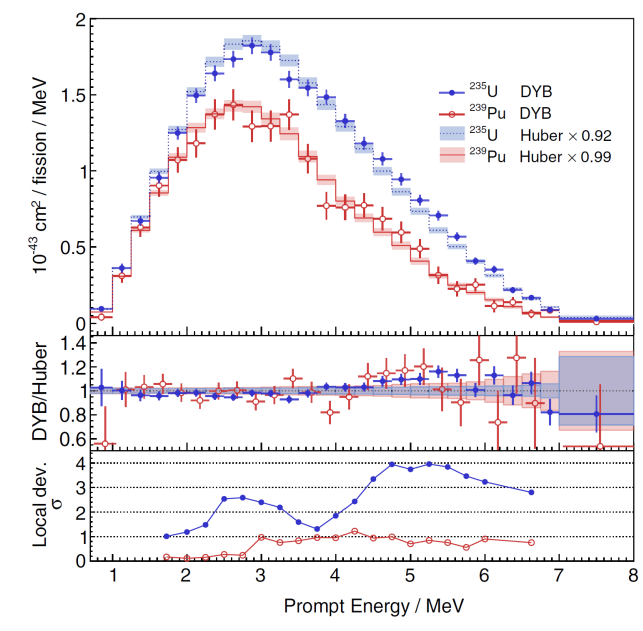

Figure 8: The extracted ${ }^{235} \mathrm{U}$ and ${ }^{239} \mathrm{Pu}$ spectra, compared with the Huber-Mueller model predictions. [9]

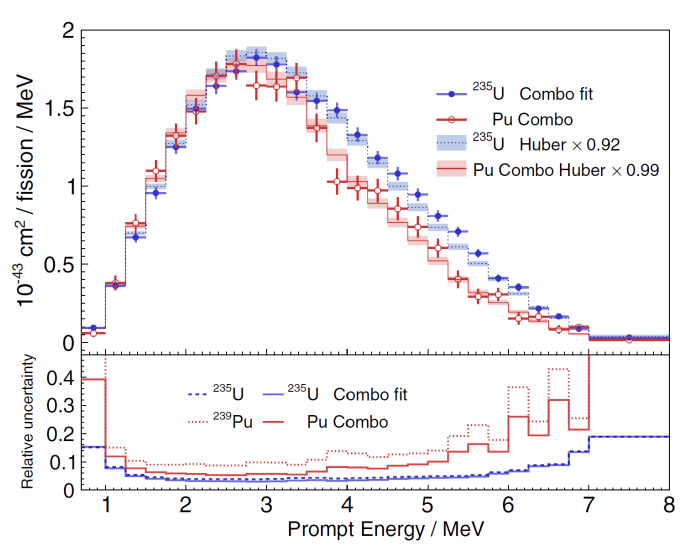

Figure 9: The extracted ${ }^{235} \mathrm{U}$ and $\mathrm{Pu}$ combo spectra compared with the Huber-Mueller model predictions. [9]

Compared with the Huber-Mueller model prediction as shown in the bottom panel of Figure 8 , the ${ }^{235} \mathrm{U}$ and ${ }^{239} \mathrm{Pu}$ spectra have similar bump structures as the total spectrum in Figure 4 in the $4 \sim 6 \mathrm{MeV}$ energy range. The local deviation for ${ }^{235} \mathrm{U}$ is $4 \sigma$ and for ${ }^{239} \mathrm{Pu}$ it is $1.2 \sigma$. The IBD yield ratio of data/prediction for ${ }^{235} \mathrm{U}$ is $0.92 \pm 0.023$ (exp.) \pm 0.021 (model) and ${ }^{239} \mathrm{Pu}$ is $0.99 \pm 0.057($ exp. $) \pm 0.025$ (model $)$. 
The extracted ${ }^{235} \mathrm{U}$ and ${ }^{239} \mathrm{Pu}$ spectra have a certain dependence on the inputs of ${ }^{238} \mathrm{U}$ and ${ }^{241} \mathrm{Pu}$ energy spectra. The fission fraction of ${ }^{241} \mathrm{Pu}$ is approximately proportional to ${ }^{239} \mathrm{Pu}$, so we combined ${ }^{239} \mathrm{Pu}$ and ${ }^{241} \mathrm{Pu}$ as one term to reduce the uncertainty dependence of $\mathrm{Pu}: s_{\text {combo }}=s_{239}+0.183 \times s_{241}$, where the coefficient 0.183, shown as the solid line in Figure 6, is the average fission fraction ratio of ${ }^{241} \mathrm{Pu}$ to ${ }^{239} \mathrm{Pu}$ in 1958 days. Figure 9 shows the extracted $\mathrm{Pu}$-combo spectrum and ${ }^{235} \mathrm{U}$ spectrum. The dependence on the input of ${ }^{241} \mathrm{Pu}$ is largely removed, and the extracted Pu-combo spectrum uncertainty is reduced to $6 \%$ from $9 \%$ in the extracted ${ }^{239} \mathrm{Pu}$ spectrum without the Pu combination.

\section{Summary}

With persistent efforts, the Daya Bay Neutrino Experiment reduces the systematic uncertainties such as energy scale, neutron detection efficiency, etc., and have acquired $>50 \%$ more statistics than the previous 621 days analysis.

The uncertainty of the measured reactor antineutrino flux is improved. The data/prediction ratio is $0.952 \pm 0.014$ (exp.) \pm 0.023 (model), and agrees with other experiments. The improved measurement of the reactor antineutrino spectrum disagrees with the Huber-Mueller model prediction in spectral shape with $5.3 \sigma$ significance in the entire energy range, and $6.3 \sigma$ in the local deviation in the $4 \sim 6 \mathrm{MeV}$ energy region. With the improved systematic uncertainty and the large statistics, the Daya Bay Neutrino Experiment made the first extraction of the ${ }^{235} \mathrm{U}$ and ${ }^{239} \mathrm{Pu}$ spectra of commercial reactors, both of the two spectrum have similar excess in the $4 \sim 6 \mathrm{MeV}$ range as that in the total spectrum.

The extracted spectrum of ${ }^{235} \mathrm{U}$ and ${ }^{239} \mathrm{Pu}$ can also be used as a reference spectra for reactor $\bar{v}_{e}$ spectrum model studies and for the $\bar{v}_{e}$ spectrum prediction of future reactor experiments .

\section{References}

[1] F. P. An et al. (Daya Bay collaboration), Phys. Rev. Lett. 108, 171803 (2012).

[2] P. Huber, Phys. Rev. C 84, 024617 (2011), 85, 029901(E) (2012).

[3] T. Mueller et al. Phys. Rev. C 83, 054615 (2011).

[4] P. Vogel, G. K. Schenter, F. M. Mann, and R. E. Schenter, Phys. Rev. C 24, 1543 (1981).

[5] F. P. An et al. (Daya Bay collaboration), Chinese Physics C 41, 013002 (2017)

[6] D. Adey et al. (Daya Bay collaboration), Phys. Rev. D 100, 052004 (2019)

[7] D. Adey et al. (Daya Bay collaboration), Nuclear Inst. and Methods in Physics Research, A 940 (2019) 230-242

[8] D. Adey et al. (Daya Bay collaboration), Phys. Rev. Lett. 121, 241805 (2018)

[9] F. P. An et al. (Daya Bay collaboration), Phys. Rev. Lett. 123, 111801 (2019)

[10] F. P. An et al. (Daya Bay collaboration), Phys. Rev. Lett. 118, 251801 (2017) 